\title{
A LOWER BOUND FOR THE NUMBER OF REIDEMEISTER MOVES FOR UNKNOTTING
}

\author{
CHUICHIRO HAYASHI
}

Department of Mathematical and Physical Sciences, Faculty of Science, Japan Women's University, 2-8-1 Mejirodai, Bunkyo-ku, Tokyo, 112-8681, Japan. hayashic@fc.jwu.ac.jp

\section{Dedicated to Professor Yukio Matsumoto for his 60th birthday. I would like to thank him for his encouragement, and letting me study anything I like when I was a student.}

\begin{abstract}
How many Reidemeister moves do we need for unknotting a given diagram of the trivial knot? Hass and Lagarias gave an upper bound. We give an upper bound for deforming a diagram of a split link to be disconnected.

On the other hand, the absolute value of the writhe gives a lower bound of the number of Reidemeister I moves for unknotting. That of a complexity of knot diagram "cowrithe" works for Reidemeister II, III moves.

We give an example of an infinite sequence of diagrams $D_{n}$ of the trivial knot with an $O(n)$ number of crossings such that the author expects the number of Reidemeister moves needed for unknotting it to be $O\left(n^{2}\right)$. However, writhe and cowrithe do not prove this.
\end{abstract}

1. An UPPER BOUND FOR THE NUMBER OF REIDEMEISTER MOVES FOR UNLINKING

A Reidemeister move is a local move of a link diagram as in Figure 1. Any such move does not change the link type. As Alexander and Briggs [1] and Reidemeister [7] showed that, for any pair of diagrams $D_{1}, D_{2}$ which represent the same link type, there is a finite sequence of Reidemeister moves which deforms $D_{1}$ to $D_{2}$.

Let $D$ be a diagram of the trivial knot. We consider sequences of Reidemeister moves which unknot $D$, i.e., deform $D$ to have no crossing. Over all such sequences, we set $u r(D)$ to be the minimal number of the moves in a sequence. Then let $u r(n)$ denote the maximum ur $(D)$ over all digrams of the trivial knot with $n$ crossings. In [3], J. Hass and J. Lagarias gave an upper bound for $\operatorname{ur}(n)$, showing that $\operatorname{ur}(n) \leq 2^{c n}$, where $c=10^{11}$. (See also [2].)

The author is partially supported by Grant-in-Aid for Scientific Research (No. 15740047), Ministry of Education, Science, Sports and Technology, Japan. 


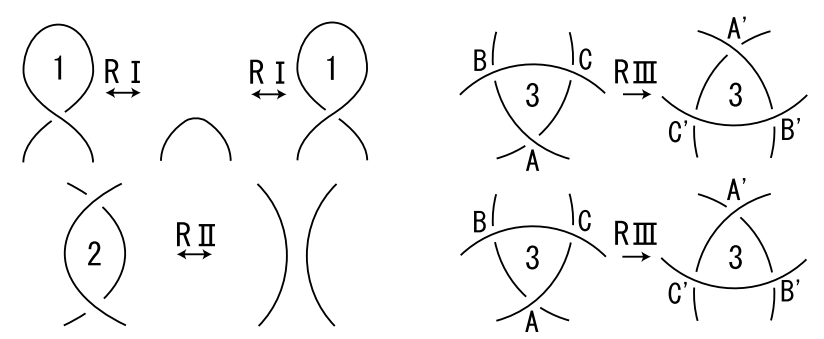

FiguRE 1

We obtain a similar upper bound for splitting links. The difficulty is that we cannot deform a link along a splitting sphere because they are disjoint.

Theorem 1.1. Let $D$ be a connected diagram of a split link with $n$ crossings. Suppose that $D$ does not have a monogon face nor a bigon face which we can apply Reidemeister II move to delete it. Assume that every component of the link has both over crossing points and under crossing points. Then there is a sequence of at most $\sum_{k=0}^{2^{128 n}} 3^{P_{k}}$ Reidemeister moves which deforms $D$ to be disconnected, where $P_{0}=2^{129 n}$ and $P_{k+1}=3^{P_{k}}$.

\section{A LOWER BOUND FOR THE NUMBER OF REIDEMEISTER MOVES FOR UNKNOTTING}

It is well-known that the absolute value of the writhe $|w(D)|$ gives a lower bound of the number of Reidemeister I moves for unknotting. We introduce a tool for estimation of the number of Reidemeister II, III moves. Let $D \subset S^{2}$ be a diagram of a knot $K \subset S^{3}$. We consider another complexity cowrithe $x(D)$. Let $C h(D)$ denote the chord diagram of $D$. That is, for an embedding $f: S^{1} \rightarrow K \subset S^{3}$ of the unit circle $S^{1} \subset \mathbb{R}^{2}$, a projection $\pi: K \rightarrow D \subset S^{2}$ and every crossing point $P$ of $D$, the two preimage points $(\pi \circ f)^{-1}(P)$ in $S^{1}$ are connected by a chord $C h_{P}$, i.e., a straight line segment in $\mathbb{R}^{2}$. The union of $S^{1}$ and the chords is $C h(D)$. Let $\operatorname{sgn}(P)$ be the sign of a crossing $P$ of $D$, for an arbitral orientation of $D$. For every pair of distinct crossings $A$ and $B$ of $D$, set $x(\{A, B\})=\operatorname{sgn}(A) \cdot \operatorname{sgn}(B)$ if the chords $C h_{A}$ and $C h_{B}$ intersect in $\mathbb{R}^{2}$. Otherwise, we set $x(\{A, B\})=0$. Then the cowrithe is determined by $x(D)=\sum x(\{A, B\})$, where the sum is taken over all the pairs of distinct crossings of $D$.

For example, let $6_{3}^{\prime}$ be the usual diagram of $6_{3}$ with the crossing $P$ changed as in Figure 2. There $x(\{P, T\})=-1, x(\{P, U\})=-1, x(\{Q, R\})=+1, x(\{Q, S\})=+1, x(\{R, S\})=$ $+1, x(\{R, T\})=-1, x(\{R, U\})=-1, x(\{S, T\})=-1, x(\{S, U\})=-1, x(\{T, U\})=+1$ and $x(\{X, Y\})=0$ for the other pairs $X, Y$. Hence $x\left(6_{3}^{\prime}\right)=-2$.

This cowrithe is a special case of $\langle A, G\rangle$ defined by M. Polyak and O. Viro in page 447 in [5], and appears in the equation in page 450 in [5] and in Corollary 4.B. on Vassiliev invariant of degree 2 in [6]. Another complexity $A_{n}$ is used in [4] for proving that the 

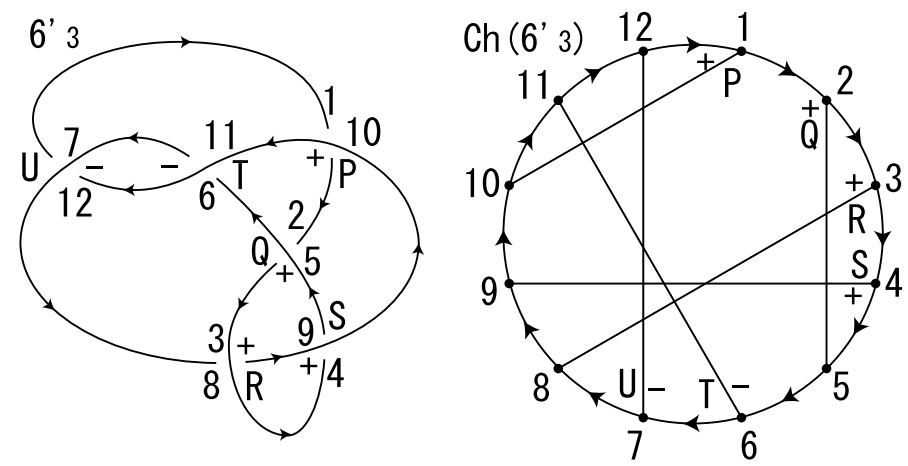

FiguRE 2

oriented usual diagram of figure 8 knot needs at least two Reidemeister III moves for being transformed into its inverse.

$x(D)$ is almost an invariant in the sense that any Reidemeister move changes it by at most a constant value.

Theorem 2.1. A Reidemeister I move does not change $x(D)$. A Reidemeister II move deleting a bigon face $f$ increases $x(D)$ by 1 if the orientations of the edges of $f$ are incoherent on the boundary circle of $f$ with respect to an orientation of D. Otherwise, it does not change $x(D)$. A Reidemeiter III move changes $x(D)$ by \pm 1 .

Corollary 2.2. Let $D$ be a diagram of the trivial knot. In any sequence of Reidemeister moves which unknots $D$, there are at least $|x(D)|$ Reidemeister II or III moves. Hence it is composed of at least $|w(D)|+|x(D)|$ Reidemeister moves.

\section{EXAMPles OF DiAgRAMS OF THE TRIVIAL KNOT}

In this appendix, we give an example of an infinite sequence of diagrams $D_{n}$ of the trivial knot with an $O(n)$ number of crossings such that the author expects the number of Reidemeister moves needed for unknotting it to be $O\left(n^{2}\right)$. However, he has no proof.
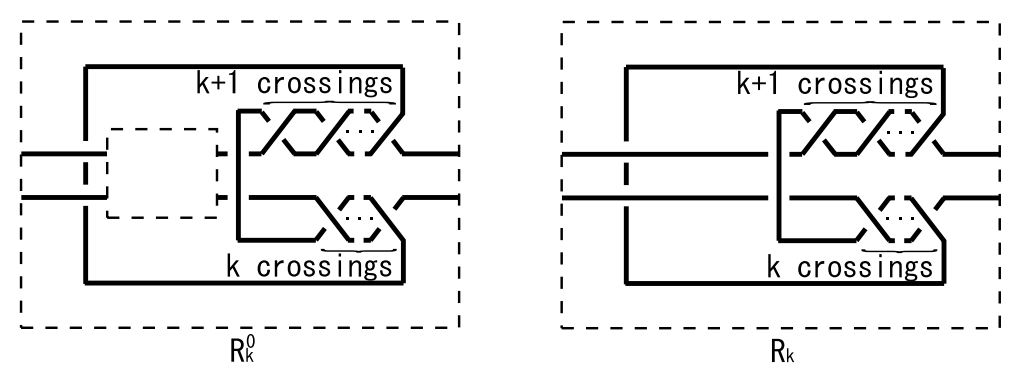

FIGURE 3

Let $R_{k}^{0}$ be a piece of a knot diagram in an annulus and $R_{k}$ another piece of knot diagram in a disc as in Figure 3, We paste $n-1$ copies of $R_{k}^{0}$ and a copy of $R_{k}$ to obtain a piece of 
a knot diagram $R_{k n}$ in a disc so that the inner boundary rectangle of the $i$-th copy of $R_{k}^{0}$ (resp. that of the $(n-1)$-st $\left.R_{k}^{0}\right)$ is glued with the outer boundary rectangle of the $(i+1)$-st copy of $R_{k}^{0}$ (resp. the boundary rectangle of $R_{k}$ ).

Let $L_{k n}$ be the mirror image of $R_{k n}$ about a vertical straight line. We glue $L_{k n}$ on the left side of $R_{k n}$ and close off the ends as in Figure 4. This diagram clearly represents the trivial knot and has $2(2 k+5) n$ crossing points. For every $k$, the author expects that the number of Reidemeister moves needed for unknotting $D_{k n}$ is $O\left(n^{2}\right)$.

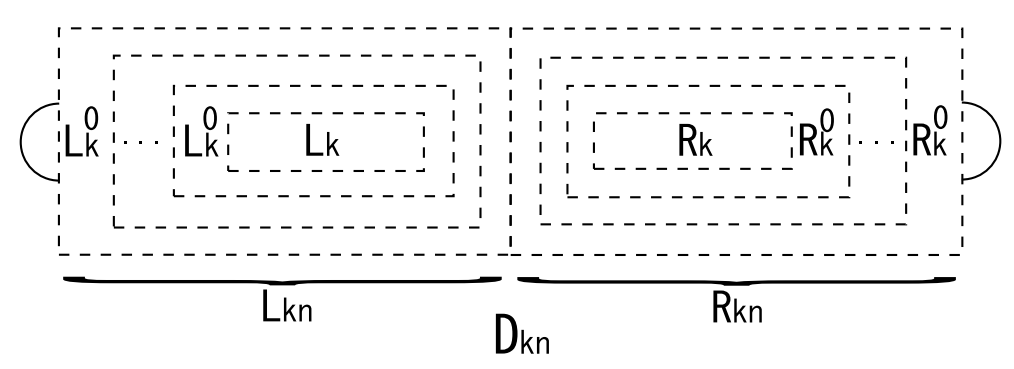

FiguRE 4

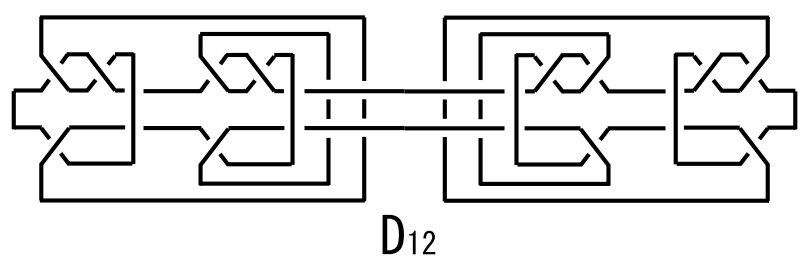

FIGURE 5

\section{REFERENCES}

1. J.W. Alexander and G.B. Briggs, On types of knotted curves. Ann. of Math. (2) 28 (1926/27), no. 1-4, $562-586$.

2. S. Galatolo, On a problem in effective knot theory. Rendiconti dell Accademia dei Lincei 9 (1999), no. 4, 299-306.

3. J. Hass and J. Lagarias, The number of Reidemeister moves needed for unknotting. J. Amer. Math. Soc. 14 (2001), no. 2, 399-428.

4. O.-P. Östlund, Invariants of knot diagrams and relations among Reidemeister moves. J. Knot Theory Ramifications 10 (2001), no. 8, 1215-1227.

5. M. Polyak and O. Viro, Gauss diagram formulas for Vassiliev invariants. Internat. Math. Res. Notices 1994, no. 11, 445-453.

6. M. Polyak and O. Viro, On the Casson knot invariant. J. Knot Theory Ramifications 10 (2001), no. 5, 711-738.

7. K. Reidemeister, Elementare Beründang der Knotentheorie. Abh. Math. Sem. Univ. Hamburg 5 (1926), $24-32$. 